\title{
Amas de lacets markoviens
}

\author{
Yves Le Jan \\ Université Paris Sud et Institut Universitaire de France.
}

June 25, 2012

\begin{abstract}
On étudie les amas définis par les ensembles poissoniens de lacets markoviens.
\end{abstract}

Abstract

We study the clusters defined by Poisson processes of Markov loops.

\section{Définitions}

La notion d'ensemble poissonnien de lacets a été introduite par Lawler et Werner dans [2] (voir aussi [5]) et les partitions associées définissent les processus CLE (voir [4]). Cette théorie profonde est basée sur l'invariance conforme. Les faits présentés ici, qui complètent le travail effectué dans [3], ont un caractère plus élémentaire mais indiquent que les ensembles poissonniens de lacets et les partitions qu'elles définissent ont un intérêt plus général.

Sur un graphe fini ou dénombrable $\mathcal{G}=(X, L)$, on supposera donné un système de conductances $C_{e}, e \in L$. On notera $C_{x, y}$ la somme des conductances des liens liant $x$ à $y$. On supposera donné de surcroît une mesure positive $\kappa_{x}$ et nous poserons $\lambda_{x}=\sum_{y} C_{x, y}+\kappa_{x}$.

Nous référons à [3] pour un exposé rapide de la théorie du potentiel et les mesures sur les espaces de trajectoires qui sont associées à ces données en nous bornant ici à rappeler quelques notations.

Nous faisons l'hypothèse que la matrice de transition $P_{y}^{x}=\frac{C_{x, y}}{\lambda_{y}}$ est transiente et nous notons $G^{x, y}$ la fonction de Green associée $\left[\left(M_{\lambda}-C\right)^{-1}\right]^{x, y}$, où $M_{\lambda}$ désigne la matrice diagonale représentant la multiplication par $\lambda$. Elle peut aussi s'écrire $\int_{0}^{\infty} p_{t}^{x, y} d t$ où $p_{t}^{x, y}=\frac{[\exp (t P)]_{y}^{x}}{\lambda_{y}}$. Nous notons $\mathbb{P}_{t}^{x, y}$ la mesure de pont de masse $p_{t}^{x, y}$ définie par la formule de Kolmogorov : pour tous $0<t_{1}<t_{2} \ldots<$ $t_{n}<t$ et $a_{i} \in X$

$$
\mathbb{P}_{t}^{x, y}\left(\xi_{t_{1}}=a_{1}, \xi_{t_{2}}=a_{2}, \ldots \xi_{t_{n}}=a_{n}\right)=p_{t_{1}}^{x, a_{1}} \lambda_{a_{1}} p_{t_{2}-t_{1}}^{a_{1}, a_{2}} \lambda_{a_{2}} \ldots p_{t-t_{n}}^{a_{n}, y}
$$

Enfin nous noterons $\mu$ la mesure sur l'espace $\mathcal{L}(X)$ des lacets sur $X$ définie par $\sum_{x} \lambda_{x} \int_{0}^{\infty} \frac{1}{t} \mathbb{P}_{t}^{x, x} d t$. La masse des lacets non triviaux (i.e. non réduits à un point) est $-\log (\operatorname{det}(I-P))$. 
On notera $\mathcal{L P}$ le processus de Poisson ponctuel d'intensité $d \alpha \mu(d l)$ défini sur $\mathbb{R}^{+} \otimes \mathcal{L}(X)$ et on posera $\mathcal{L}_{\alpha}=\mathcal{L} \mathcal{P} \cap[0 \alpha] \times \mathcal{L}(X)$, définissant ainsi une famille d'ensemble poissoniens de lacets croissante et à accroissements indépendants stationnaires.

Posons $x \sim_{\alpha} y$ si et seulement si il existe une famille finie $\left(l_{1} \ldots l_{m}\right)$ de lacets de $\mathcal{L}_{\alpha}$, telle que $x \in l_{1}, y \in l_{2}$ et $l_{i} \cap l_{i+1} \neq \emptyset$ pour tout $1 \leq i<m$. On note $\mathcal{C}_{\alpha}$ la famille décroissante de partitions associées avec $\mathcal{C}_{0}=\{\{x\}, x \in X\}$ et $\mathcal{C}_{\infty}=\{X\}$.

Toute partition $\mathcal{D}$ de $X$ définit un graphe quotient $\mathcal{G} / \mathcal{D}$ dont les sommets sont les éléments de $\mathcal{D}$ et les liens les éléments de $L$ qui relient deux parties distinctes.

\section{Quelques résultats généraux}

Etant donnée une partition finie ou dénombrable $\mathcal{D}=\left\{D_{i}\right\}$ de $X$, il est facile ( Cf [3] 4-3) de calculer la probabilité pour que $\mathcal{C}_{\alpha}$ soit plus fine que $\mathcal{D}$ :

$$
\mathbb{P}\left(\mathcal{C}_{\alpha} \succeq \mathcal{D}\right)=\left(\frac{\prod \operatorname{det}\left(G_{D_{i}}\right)}{\operatorname{det}(G)}\right)^{\alpha}
$$

(où $G_{D}$ désigne la fonction de Green de $D$, c'est à dire l'inverse de la restriction à $D$ de $\left.M_{\lambda}-C\right)$.

Ces probabilités déterminent la loi de $\mathcal{C}_{\alpha}$. On a notamment, en notant $\mathfrak{P}_{k}$ la famille des partitions de $X$ en $k$ parties non vides :

$\mathbb{P}\left(\mathcal{C}_{\alpha}=\{X\}\right)=1-\sum_{\Pi_{2} \in \mathfrak{P}_{2}} \mathbb{P}\left(\mathcal{C}_{\alpha} \succeq \Pi_{2}\right)+\ldots+(-1)^{k-1}(k-1) ! \sum_{\Pi_{k} \in \mathfrak{P}_{k}} \mathbb{P}\left(\mathcal{C}_{\alpha} \succeq \Pi_{k}\right)-\ldots$

Si l'on part d'une partition $\mathcal{D}$ plus fine qu'une autre partition $\mathcal{D}^{\prime}$, on a une formule analogue pour la probabilité de transition en temps $\alpha$ de $\mathcal{D}$ vers $\mathcal{D}^{\prime}$.

Par ailleurs, la loi de $\mathcal{C}_{\alpha}$ est invariante par $h$-transformation (cf: [3] p. 60 Remarque 10).

Si $D$ est une partie de $X$, la partition de $D$ induite par $\mathcal{L}_{\alpha}^{D}$ (les lacets de $\mathcal{L}_{\alpha}$ contenus dans $D$ ), notée $\mathcal{C}_{\alpha}^{D}$ est en général plus fine que la restriction de $\mathcal{C}_{\alpha}$ à $D$ mais elle coïncide avec elle lorsque $D$ est réunion d'atomes de $\mathcal{C}_{\alpha}$. En particulier,

$$
\mathbb{P}\left(\mathcal{C}_{\alpha}=\mathcal{D}\right)=\mathbb{P}\left(\mathcal{C}_{\alpha} \succeq \mathcal{D}\right) \prod_{i} \mathbb{P}\left(\mathcal{C}_{\alpha}^{D_{i}}=\left\{D_{i}\right\}\right)
$$

Notons aussi que si $U \subset D \subset X$,

$$
\mathbb{P}\left(\mathcal{C}_{\alpha} \succeq\{U, X-U\}\right)=\mathbb{P}\left(\mathcal{C}_{\alpha}^{D} \succeq\{U, D-U\}\right) \mathbb{P}\left(\nexists l \in \mathcal{L}_{\alpha} \text { visitant } U \text { et } X-D\right)
$$

le deuxième terme du deuxième membre étant égal à $\left(\frac{\operatorname{det}\left(G_{X-U}\right) \operatorname{det}\left(G_{D}\right)}{\operatorname{det}(G) \operatorname{det}\left(G_{D-U}\right)}\right)^{\alpha}=$ $\left(\frac{\operatorname{det}(G)_{\mid U \times U} \operatorname{det}(G)_{\mid X-D \times X-D}}{\operatorname{det}(G)_{\mid((U \cup X)-D) \times((U \cup X)-D)}}\right)^{-\alpha}$. 
Notons enfin que si $K$ est un ensemble de liens de $L$ liant des éléments distincts de $\mathcal{D}$, en notant $N_{e}^{(\alpha)}$ le nombre total de sauts des lacets de $\mathcal{L}_{\alpha}$ à travers le lien $e$,

$$
\mathbb{P}\left(\mathcal{C}_{\alpha} \succeq \mathcal{D} \mid N_{e}^{(\alpha)}=0 \forall e \in K\right)=\left(\frac{\prod \operatorname{det}\left(G_{D_{i}}\right)}{\operatorname{det}\left(G^{(K)}\right)}\right)^{\alpha}
$$

où $G^{(K)}$ désigne la fonction de Green obtenue en annulant les conductances des liens de $K$ et en augmentant $\kappa$ en leurs extémités de manière à ce que $\lambda$ ne change pas.

L'évolution en $\alpha$ de $\mathcal{C}_{\alpha}$ est markovienne. Le taux de transition de $\mathcal{D}$ vers la partition dans laquelle les parties $\left\{D_{j}, j \in J\right\}$ sont réunies est

$$
\begin{aligned}
& \log \left(\operatorname{det}\left(G_{\cup_{j \in J} D_{j}}\right)\right)-\sum_{j_{1} \in J} \log \left(\operatorname{det}\left(G_{\cup_{j \neq j_{1} \in J} D_{j}}\right)\right) \\
& \ldots+\sum_{j_{1} \neq j_{2} \in J} \log \left(\operatorname{det}\left(G_{\cup_{j \neq j_{1}, j_{2} \in J} D_{j}}\right)\right) \ldots+(-1)^{J-1} \sum_{j \in J} \log \left(\operatorname{det}\left(G_{D_{j}}\right)\right)
\end{aligned}
$$

On peut donner une autre expression de $\mathbb{P}\left(\mathcal{C}_{\alpha} \succeq \mathcal{D}\right)$. Elle s'écrit aussi $\operatorname{det}\left(K_{\mathcal{D}}\right)^{\alpha}$, avec $K_{\mathcal{D}}=\left(\otimes G_{D_{i}}\right) G^{-1}$. La restriction de $\bar{K}_{\mathcal{D}}$ à $D_{i} \times D_{j}$ est l'identité si $i=j$ et moins la restriction à $D_{j}$ de la distribution de sortie de $D_{i}$ si $i \neq j$. Notons que cette dernière sous-matrice est nulle s'il n'y a pas de lien direct entre $D_{i}$ et $D_{j}$. Notons aussi que si $M$ est la réunion des points frontières des $D_{i}$, il suffit de considérer la trace sur $M$ de l'ensemble de lacets et de la partition. Le calcul peut alors être beaucoup plus simple, notamment dans le cas unidimensionnel.

Il existe une relation intéressante avec le champ libre dans le cas où $\alpha$ est un demi entier. Si $\mathcal{D}$ est une partition, posons $C_{e}^{\prime}=C_{e} 1_{\{e \in L(\mathcal{D})\}}$ et $\kappa^{\prime}=$ $\kappa+\sum_{e=\{x, y\} \in L(\mathcal{D})} C_{e}$. D'après [3], p. 66, on a $\mathbb{P}\left(\mathcal{C}_{\frac{1}{2}} \succeq \mathcal{D}\right)=\left(\frac{\operatorname{det}\left(G^{\prime}\right)}{\operatorname{det}(G)}\right)^{\frac{1}{2}}=$ $\mathbb{E}\left(e^{-\sum_{\{x, y\} \in L(\mathcal{D})} C_{x y} \phi(x) \phi(y)}\right)$ avec $G^{\prime}=\left(M_{\lambda}-C^{\prime}\right)^{-1}$

\section{Percolation}

Les amas de percolation dans $(X, L)$ apparaissent comme un cas limite de partition en amas de lacets, dans lequel les seuls les lacets faisant un aller-retour entre deux points contribuent. En effet, si pour simplifier nous supposons $X$ fini , les conductances égales à l'unité et faisons varier les paramètres $\kappa$ et $\lambda$, lorsque $\kappa_{n} \uparrow \infty$ et $\alpha_{n} \sim u \kappa_{n}^{2}$, avec $u>0, \mathcal{C}_{\alpha_{n}}$ converge en loi vers la partition en amas $\mathcal{P}_{u}$ induite par la percolation de paramètre $1-e^{-u}$. De fait, cette dernière est caractérisée par les identités:

$$
\mathbb{P}\left(\mathcal{P}_{u} \succeq \mathcal{D}\right)=e^{-u|L(\mathcal{D})|}
$$

où $L(\mathcal{D})$ désigne l'ensemble des liens de $L$ qui lient deux éléments distincts de $\mathcal{D}$ et on note que $\left[K_{\mathcal{D}}\right]_{x, y} \sim \kappa_{n}^{-1}$ si $\{x, y\}$ appartient à $L(\mathcal{D})$ et est d'ordre inférieur sinon. Alors, $\log \left(\operatorname{det}\left(K_{\mathcal{D}}\right)\right)=\operatorname{Tr}\left(\log \left(K_{\mathcal{D}}\right)\right)$ est équivalent à $-\frac{1}{2} \operatorname{Tr}\left(Q^{2}\right)$, où $Q_{x, y}=\kappa_{n}^{-1} 1_{\{\{x, y\} \in L(\mathcal{D})\}}$. 


\section{Fragmentation}

On peut définir un processus de fragmentation allant de $\{X\}$ à $\mathcal{C}_{\alpha}$ à partir du précédent en retournant le temps à partir du temps de coalescence final. Les taux de fragmentation de $A$ en $\left\{A_{i}\right\}$ sont alors identiques aux taux de coalescence de $\left\{A_{i}\right\}$ en $A$.

Le processus de fragmentation peut être défini à partir d'une partition quelconque en fragmentant indépendamment chaque partie. On peut aussi contruire la fragmentation à partir de l'infini en effaçant progressivement les lacets. Enfin, on peut construire des partitions fluctuantes en mélangeant coalescence et fragmentation. Ces processus peuvent être construits à partir d'un processus de Poisson de lacets marqués par un temps de naissance et une durée de vie exponentielle.

\section{Quelques exemples}

a) Si $\mathcal{G}$ est le graphe complet de taille $N$, avec des conductances égales à 1 et la mesure $\kappa$ constante, on a

$$
\mathbb{P}\left(\mathcal{C}_{\alpha} \succeq \mathcal{D}\right)=\left(1-\frac{N}{N+\kappa}\right)^{\alpha} \prod\left(1-\frac{\left|D_{i}\right|}{N+\kappa}\right)^{-\alpha}
$$

Notons que $\left(1-\frac{j}{N+\kappa}\right)^{\alpha}$ est le $j$-ième moment $m_{j}$ de $\exp \left(\frac{Z}{N+\kappa}\right)$ où $Z$ est une variable de loi $\Gamma(\alpha)$, de sorte que $\mathbb{P}\left(\mathcal{C}_{\alpha} \succeq \mathcal{D}\right)=\frac{m_{N}}{\left.\prod^{m}\right|_{i} \mid}$ et que $\mathbb{P}\left(\mathcal{C}_{\alpha}=\mathcal{C}_{\infty}\right)=\frac{c_{N}}{m_{N}}$ où $c_{N}$ désigne le $n$-ième cumulant de $Z$.

b) Dans le cas de la marche aléatoire simple tuée en zéro, les liens vides séparant les amas forment des processus de renouvellement $\left(S_{n}^{(\alpha)}, n>0\right)$ dont les lois ont pour fonction génératrice $1-\frac{s}{L i_{\alpha}(s)}(L i$ désignant le polylogarithme). Pour $\alpha>1, \mathbb{P}\left(S_{1}^{(\alpha)}=\infty\right)=\frac{1}{\zeta(\alpha)}$. Les graphes quotients sont toujours isomorphes à $\mathbb{N}$. Pour $\alpha<1$, lorsque $\varepsilon$ tend vers $0,\left(\varepsilon S_{\left[\varepsilon^{\alpha-1} t\right]}^{(\alpha)}, t \geq 0\right)$ converge en loi vers un subordinateur stable $\left(S_{t}^{(\alpha)}, t \geq 0\right)$ d'indice $1-\alpha$. On a un résultat analogue pour la marche aléatoire sur $\mathbb{Z}$ tuée à taux constant. Dans le cas d'un intervalle fini $\left[\begin{array}{ll}0 & L\end{array}\right]$, on obtient un processus de renouvellement conditionné à sauter en $L$. Les graphes quotients sont toujours des intervalles finis.

c) Dans le cas limite du mouvement brownien, les amas de lacets sont donnés par les sauts de subordinateurs, ou sur un intervalle fini, par des sauts de ponts de subordinateurs. De plus, il résulte de la formule de composition des subordinateurs que la partition $\mathcal{C}_{\alpha}$ sur l'espace quotient par $\mathcal{C}_{\beta}$ définit une partition identique en loi à $\mathcal{C}_{\alpha+\beta-\alpha \beta}$. Du fait de l'échangeabilité des sauts, ceci induit un processus de coalescence de type classique, le coalescent de Bolthausen Sznitman (Cf [1]). Le processus de fragmentation inverse est le processus de branchement continu de Neveu. 


\section{Lacets de liens orientés et réduction}

Soit $L^{O}$ l'ensemble des liens orientés. Etant donné un lien orienté $e$, nous notons $e^{-}$son origine, $e^{+}$son extrémité et $e^{*}$ le lien de sens opposé. Notons $Q$ la matrice de transition sur $L^{O}$ définie par $Q_{e_{1} e_{2}}=P_{e_{2}^{-}}^{e^{+}}, W$ l'inverse de $I-Q$ et $\mu^{O}$ la mesure associée sur les lacets formés de liens orientés. $\mu^{O}$ se projette naturellement sur la restriction de $\mu$ aux lacets non triviaux. On notera $\mathcal{L}_{\alpha}^{O}$ et $\mathcal{C}_{\alpha}^{O}$ les ensembles poissonniens de lacets et les amas ainsi définis dans $L^{O}$. Beaucoup de résultats établis dans le cadre défini précédemment s'étendent. On a notamment $\mathbb{P}\left(e \in \mathcal{L}_{\alpha}^{O}\right)=\left[W_{e e}\right]^{-\alpha}$ et

$$
\log (\operatorname{det}(I-P))=\mu(\text { lacets non triviaux })=\mu^{O}(1)=\log (\operatorname{det}(I-Q))
$$

On en déduit une autre expression pour la loi de $\mathcal{C}_{\alpha}$ :

$$
\mathbb{P}\left(\mathcal{C}_{\alpha} \succeq \mathcal{D}\right)=\left(\frac{\operatorname{det}\left(I-Q_{\mid L-L(\mathcal{D})}\right)}{\operatorname{det}(I-Q)}\right)^{-\alpha}=\operatorname{det}\left(W_{\mid L(\mathcal{D})}\right)^{\alpha}
$$

Un des intérêts de cette définition réside dans le bon comportement de $\mathcal{L}_{\alpha}^{O}$ quand on réduit le graphe. La réduction $\mathcal{G} \rightarrow \mathcal{G}^{(e)}$ est effectuée en supprimant un lien $e$ et en identifiant ses extrémités (les autres liens qui les lient deviennent des boucles). La matrice de transition $Q$ est modifiée comme suit : $\mathrm{Si}_{1}^{+}=$ $e^{-}$et $e_{2}^{-}=e^{+}, Q_{e_{1} e_{2}}^{(e)}=c_{e} Q_{e_{1} e} Q_{e e_{2}}$ avec $c_{e}=\frac{1}{1-Q_{e e^{*} Q_{e^{*}}}}$, Si $e_{1}^{+}=e^{-}$et $e_{2}^{-}=e^{-}, Q_{e_{1} e_{2}}^{(e)}=c_{e} Q_{e_{1} e_{2}}$, avec des formules analogues pour les cas $e_{1}^{+}=e^{+}$, $e_{2}^{-}=e^{-}$et $e_{1}^{+}=e^{+}, e_{2}^{-}=e^{+}$. Les autres probabilités de transition ne sont pas modifiées. L'image de $\mathcal{L}_{\alpha}^{O}$ dans le graphe réduit a la même loi que le $\mathcal{L}_{\alpha}^{O}$ défini $\operatorname{par} Q^{(e)}$ sur le graphe préalablement réduit. Ce n'est pas le cas pour $\mathcal{L}_{\alpha}$.

\section{Plongement combinatoire}

Considérons un plongement combinatoire de $\mathcal{G}$, c'est à dire la donnée, en chaque point, d'un ordre circulaire sur les liens orientés qui en sont issus. (ceci permet de plonger le graphe dans une surface). Si $e$ est un lien orienté, on note $\tau e$ le lien suivant parmi les liens issus de son origine $e^{-}$. En itérant $\theta: e \rightarrow-\tau e$, on définit des faces qui sont les orbites de $\theta$. On note $\mathcal{G}^{*, \tau}$ le graphe dual dont les sommets sont les faces. Un lien existe entre deux sommets si les deux faces correspondantes sont adjacentes. Les éléments de $L$ sont donc associés à des liens de $\mathcal{G}^{*, \tau}$, avec la possibilité de boucles liant un sommet-face à lui même.

A toute partition on associe le sous graphe séparateur $\mathcal{G}_{\mathcal{D}}^{*, \tau}$ de $\mathcal{G}^{*, \tau}$ formés par les faces adjacentes à plus d'un élément de $\mathcal{D}$ et les liens de $L(\mathcal{D})$. Le processus $\mathcal{G}_{\mathcal{C}_{\alpha}}^{*, \tau}$ définit ainsi un arbre aléatoire décroissant de sous graphes connexes inclus les uns dans les autres et on a :

$$
\mathbb{P}\left(\mathcal{G}_{\mathcal{C}_{\alpha}}^{*, \tau} \supseteq \mathcal{G}_{\mathcal{D}}^{*, \tau}\right)=\operatorname{det}\left(W_{\mid L(\mathcal{D})}\right)^{\alpha}
$$


Le plongement $\tau$ permet aussi de définir des arbres d'exploration de $L(\mathcal{D})$ : à tout lien orienté $e$ on associe $\left\{-\left(\theta^{k}\right)(e), k \in\left\{n,\left(\theta^{n}\right)(e) \in L(\mathcal{D})\right\}\right\}$. Ce processus est particulièrement simple dans le cas des triangulations.

\section{References}

[1] Bertoin J., Pitman J. Two coalescents derived from the ranges of stable subordinators. EJPECP 5 (7) 1-17 (2000)

[2] Lawler G., Werner W. The Brownian loop soup. PTRF 128 565-588 (2004).

[3] Le Jan Y. Markov paths, loops and fields. LNM 2026 Springer (2011)

[4] Sheffield S.,Werner W. Conformal loop ensembles: the Markovian characterization and the loop-soup construction. ArXiv 10062374 (2010). To appear in Ann. Maths.

[5] Symanzik K. Euclidean quantum field theory. Scuola internazionale di Fisica "Enrico Fermi". XLV Corso. 152-223 Academic Press (1969). 